\title{
Reflets
}

Revue ontaroise d'intervention sociale et communautaire

\section{Le projet de loi 96 et ses répercussions}

\section{Derrick Barnes}

Volume 4, numéro 1, printemps 1998

Intervention en contextes minoritaires

URI : https://id.erudit.org/iderudit/026210ar

DOI : https://doi.org/10.7202/026210ar

Aller au sommaire du numéro

Éditeur(s)

Reflets : Revue ontaroise d'intervention sociale et communautaire

ISSN

1203-4576 (imprimé)

1712-8498 (numérique)

Découvrir la revue

Citer cet article

Barnes, D. (1998). Le projet de loi 96 et ses répercussions. Reflets, 4(1), 210-216.

https://doi.org/10.7202/026210ar

Tous droits réservés (C) Reflets : Revue ontaroise d'intervention sociale et communautaire, 1998

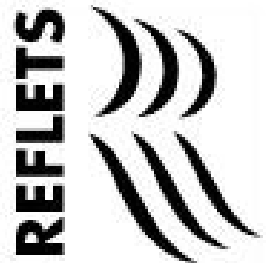

Ce document est protégé par la loi sur le droit d'auteur. L’utilisation des services d’Érudit (y compris la reproduction) est assujettie à sa politique d'utilisation que vous pouvez consulter en ligne.

https://apropos.erudit.org/fr/usagers/politique-dutilisation/ 


\title{
Le projet de loi 96 et ses répercussions
}

\author{
Derrick Barnes \\ Étudiant à la maîtrise en service social, Université d'Ottawa
}

Le Projet de loi 96 présenté par le gouvernement Harris modifiera les enjeux pour les locataires de l'Ontario. Cette loi, nommée Loi codifiant et révisant le droit de la location à usage d'habitation (mieux connue sous Loi sur la protection des locataires) supprime ou modifie dix-huit lois et englobe tout ce qui était traité dans la Loi sur la location immobilière, la Loi sur le contrôle des loyers et la Loi sur la protection des logements locatifs de l'Ontario. Le nouveau projet de loi a reçu la sanction royale le 28 novembre 1997 et entre en vigueur le $1^{\text {er }}$ avril 1998. En ce moment, ce projet de loi génère beaucoup de confusion quant à ses effets sur le marché locatif. Cet article vise à clarifier quelques-uns des effets possibles.

Après avoir lu plusieurs textes sur cette nouvelle loi, il me semble important d'aborder trois grands thèmes: l'abolition du contrôle des loyers; la réduction du nombre de logements à louer et les changements au Code des droits de la personne. Quoique cette loi mérite une analyse plus détaillée, les contraintes d'espace me forcent à me limiter à ces trois sujets. Si vous désirez plus de renseignements, vous trouverez une liste de contacts à la fin de cet article.

\section{Abolition du contrôle des loyers}

Le premier thème, l'abolition du contrôle du loyer, est celui qui aura probablement le plus d'impact sur le marché locatif. Selon le nouveau projet de loi, un propriétaire aura carte blanche pour fixer le coût du loyer lors d'une nouvelle location. Un appartement 
qui coûtait déjà $500 \$$ par mois pourrait donc coûter $1000 \$$ ou plus par mois pour une nouvelle locataire. Dans le système actuel, datant de 1975, c'est l'appartement qui est indexé, ce qui veut dire que peu importe le va-et-vient des locataires, le loyer ne peut augmenter qu'une seule fois par année et ce, selon le guide établi par le Bureau de contrôle des loyers (il existe des exceptions à cette règle, particulièrement lorsqu'il s'agit d'édifices dans lesquels des réparations significatives ont été faites). Sous la nouvelle loi, il va sans dire que les propriétaires seront tentés d'exiger plus d'argent pour des unités qui n'ont fait l'objet d'aucune réparation. Par contre, lorsqu'une unité est déjà louée, les augmentations annuelles des loyers continueront d'être limitées selon la norme établie par le ministère des Affaires municipales et du Logement (cette année, la norme est de 3,0 \%). Et si cela n'avantage pas assez les propriétaires, sous la nouvelle loi, une personne n'aura qu'un an pour porter plainte devant le tribunal pour un loyer illégal, ce qui représente un changement important par rapport aux six ans permis dans le système actuel. Les représentants des locataires craignent que, pour assurer un accroissement de leurs profits, les propriétaires soient tentés «d'encourager» les locataires actuels à déménager.

Le projet de loi 96 interdit le harcèlement des locataires, mais plusieurs personnes qui pourraient avoir de la difficulté à se protéger risquent néanmoins d'être expulsées. Je pense surtout à toute personne qui est en position défavorable de négociation (familles monoparentales, personnes à faible revenu ou qui manquent d'expérience de négociation). De plus, il peut être difficile de prouver ce harcèlement, qui peut prendre des formes subtiles. Ensuite, si une locataire se fait expulser, où ira-t-elle? Les loyers, qui sont déjà dispendieux dans la plupart des cas, seront augmentés et se trouveront probablement au-delà des limites financières de plusieurs personnes.

Cette augmentation des loyers présente d'autres ramifications. Premièrement, si une locataire se trouve un logement à prix abordable, elle sera probablement moins tentée d'exiger de son propriétaire des réparations nécessaires de peur de se retrouver sans logement. Il est, bien sûr, strictement défendu d'expulser une personne à cause d'une plainte, mais le propriétaire pourrait 
toujours trouver d'autres raisons pour faire pression pour qu'elle déménage de "sa propre volonté». Deuxièmement, si les locataires payent davantage pour leur loyer, ils risquent d'avoir des revenus insuffisants pour d'autres besoins essentiels tels que la nourriture ou les vêtements. En Ontario, la baisse de $21 \%$ de l'assistance sociale a déjà causé - et cause toujours - des difficultés pour les familles et les personnes pauvres. Déjà en octobre 1997, 1380 personnes de plus que l'année précédente cherchaient un refuge dans des abris à Toronto, ce qui représente une augmentation d'environ $67 \%$ depuis 1996. (Toronto Star, le 9 octobre 1997). Au lieu d'aider les personnes dans le besoin, le gouvernement Harris donne aux mieux nantis, comme l'a dit J. D. Hulchanski dans sa présentation au comité gouvernemental sur ce projet de loi (26 juin 1997) : "If only 20\% of Ontario's 1.3 million renter households pay an extra $\$ 50$ per month as a result of this legislation, a transfer of $\$ 156$ million from tenants to landlords will take place».

Le gouvernement Harris, dans Nouvelles Directions (où se trouve sa présentation initiale de ce projet de loi), a suggéré une plus grande marge de manoeuvre au marché locatif pour faciliter la construction de nouveaux logements; les propriétaires, quant à eux, disent ne plus construire de logements à cause du trop grand nombre de règlements en Ontario. Cependant, il existe des alternatives à ce projet de loi pour assurer un marché locatif rentable et en croissance. Hulchanski cite un rapport récent qui suggère, parmi d'autres méthodes, de réduire les impôts fonciers et/ou les frais municipaux, d'offrir une deuxième hypothèque sans intérêt ou une garantie d'hypothèque, des délais de paiement pour les terrains, etc. Toutes ces alternatives exigent un engagement de la part du gouvernement, qui, lui, ne semble pas prêt à le prendre. Au lieu de s'engager à améliorer le lot des locataires, le gouvernement délègue les coûts à ceux et celles qui n'ont pas les moyens de les défrayer. Cependant, ce fardeau sur les moyens limités des locataires ne garantit aucun nouveau développement. 


\section{La réduction du nombre de logements à louer}

Quant au deuxième enjeu, soit celui de la réduction du nombre de logements à louer, non seulement verrons-nous une augmentation élevée des loyers, mais nous verrons aussi une augmentation de la conversion d'appartements à d'autres usages. La loi actuelle sur la protection des logements locatifs de l'Ontario restreint la conversion des unités à louer; la règle générale était qu'avant de pouvoir autoriser la conversion d'édifices, une ville de plus de 50000 habitants devait s'assurer de remplacer les unités perdues, maintenant ainsi son parc de logements locatifs. Après la mise en vigueur du projet de loi 96, les propriétaires ne seront plus limités dans les types de conversion qu'ils pourront faire. Les condominiums et les maisons à vendre étant plus rentables que les appartements et les maisons à louer, il est possible que les conversions se fassent à un taux accéléré.

\section{Changement au Code des droits de la personne}

Le troisième enjeu a trait aux changements apportés au Code des droits de la personne. Selon le projet de loi 96, un propriétaire a le droit d'établir des critères de revenu minimum pour louer un logement. Et si le locataire mentait à propos de son revenu? Selon ce projet de loi, cela justifierait son expulsion. Cet enjeu a été soumis à la Commission des droits de la personne, mais le gouvernement Harris prend un raccourci en changeant le Code pour l'adapter aux voeux des propriétaires. Ce qui est sousentendu, c'est qu'un propriétaire est plus apte à déterminer ce que son locataire peut - ou ne peut pas - se permettre de débourser pour son loyer. En plus de l'information sur le revenu, les propriétaires pourront exiger des vérifications de crédit et des «références en matière de logement». Combien de fois verronsnous des difficultés de location liées aux critères précédents? Une 
femme de 50 ans récemment séparée n'aura pas de références de crédit si son mari a toujours assumé la charge de leurs dépenses; une famille récemment immigrée n'aura pas de dossier de crédit ni de références ou encore, un propriétaire pourra donner de mauvaises références basées sur ses préjugés. Cette loi n'a que des répercussions mineures pour les mieux nantis qui, de toute façon, sont plus susceptibles de pouvoir se payer un logement adéquat. Mais grosso modo, cette loi permet et aide à légitimer la discrimination envers les locataires.

\section{Les groupes qui seront touchés}

Ce ne sont pas tous les locataires qui subiront les même conséquences. Les populations doublement à risque sont les groupes minoritaires ou ceux qui n'ont pas les moyens de se défendre face aux changements prévus. Qu'est-ce que cette loi fera aux familles et personnes récemment immigrées? Une possibilité est claire: les propriétaires auront la possibilité de justifier leur refus de loger une famille immigrante ou n'importe quelle personne qui, sans référence de crédit, pourra ainsi se voir refuser l'accès d'un logis. De plus, ce qui est sous-entendu dans le Projet de loi 96, c'est que la négociation fera partie intégrale de toute location, propriétaire et locataire devant s'entendre sur le loyer. Une personne arrivant au Canada, ou même une personne déjà établie au Canada, ne se sentira pas nécessairement à l'aise de négocier le loyer, particulièrement lorsqu'elle ne sait pas qu'elle en a le droit ou si elle ne connait pas le loyer normal pour un appartement. Auparavant, le registre des loyers, géré par le Bureau du contrôle des loyers, donnait de l'information au sujet de locations raisonnables et légales. Maintenant, les propriétaires ne doivent plus adhérer à cette norme établie lors d'une nouvelle location. Ce qui veut dire que les personnes immigrantes et toute autre personne dans une situation désavantageuse peuvent s'attendre à une augmentation du coût du loyer. 
Pour ce qui est des groupes déjà établis, verrons-nous moins d'effets néfastes? La réponse est non. Un danger qui menace les groupes est la démolition de leurs quartiers. Le renouvellement urbain des années 1960 à 1970 a provoqué la destruction de plusieurs quartiers ethniques, répandant leurs habitants à travers la ville et détruisant le tissu social. Au lieu de valoriser les différences et les caractères uniques de ces quartiers, les spéculateurs les identifiaient comme ghettos. La Basse-ville d'Ottawa en est un très bon exemple. Ce quartier qui était à $80 \%$ franco-ontarien, ne contenait plus, après son «renouvellement», que $40 \%$ de francophones, voyant ainsi se détruire son tissu social (Welch 1997: 125). Mon hypothèse est que plusieurs quartiers risquent de revivre le même phénomène avec l'abolition de la Loi sur la protection des logements locatifs. Les spéculateurs pourraient facilement changer toute la dynamique d'un quartier lorsqu'elle ne coïncidera pas avec certains idéaux de race blanche et de classe moyenne. Il est possible que cela n'arrivera pas, mais comme on dit, l'histoire se répète souvent.

\section{Les droits qui restent...}

Pour terminer, mentionnons certains droits qui ont été préservés dans le nouveau projet de loi. Par exemple, les locataires pourront entamer des poursuites contre leur propriétaire pour obtenir une réduction de loyer si leur logement manque d'entretien; le droit au maintien dans les lieux existe toujours; les propriétaires n'ont pas le droit de demander de dépôt de garantie sauf celui du dernier mois. Et selon Dan McIntyre de la Fédération des associations de locataires d'Ottawa-Carleton, comme avec n'importe quel changement d'administration, la possibilité existe que le nouveau Tribunal du logement de l'Ontario soit moins intimidant pour les locataires, plus efficace, plus rapide et moins coûteux que le processus actuel à la Cour générale de l'Ontario et au Bureau du contrôle des loyers. Mais, comme McIntyre l'a bien noté, cela reste à voir. Une amélioration claire consiste en la précision des termes dans la nouvelle loi et la combinaison des trois lois actuelles 
pouvant aider à combler les lacunes légales et à réduire la confusion. Ceci peut être très important pour les juges, avocats et propriétaires mais n'aura sans doute pas de grandes répercussions sur les locataires qui devront subir les effets néfastes de ce projet de loi.

En guise de conclusion, il faut nous préparer à une augmentation des problèmes de logement et nous renseigner sur les changements contenus dans ce projet de loi. Cet article n'a touché qu'aux thèmes qui me semblaient les plus importants mais ce projet de loi va sans doute avoir d'autres répercussions que celles que nous avons évoquées ici.

Pour obtenir plus d'information vous pouvez communiquer avec:

- La Fédération des associations des locataires d'OttawaCarleton: (613) 247-7588

(http://www.ncf.carleton.ca/focta)

- La Coalition franco-ontarienne sur le logement: (613) $834-5911$

- Federation of Metro Tenants' Associations : (416) 921-9494

- Hamilton Area Coalition of Tenants' Associations : (905) 527-5574

- Waterloo Region Tenants' Coalition : (519) 744-3032

\section{Bibliographie}

WELCH, David (1997). «The Franco-Ontarian Community Under the Harris CounterRevolution», dans Diana Ralph, André Régimbald et Nérée St-Amand (eds), Open for Business, Closed to People, Halifax, Fernwood, 122-133. 